\title{
2011: ein Jahr voller wichtiger politischer Entscheide!
}

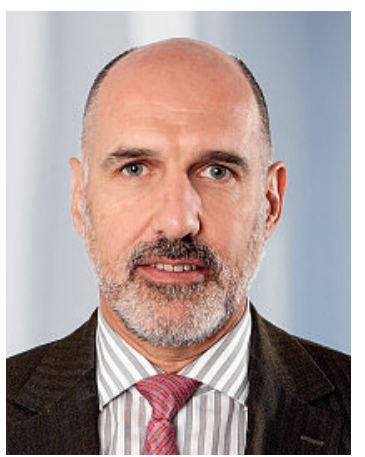

Während das Ende eines Jahres ein geeigneter Zeitpunkt ist, Bilanz zu ziehen, bietet sich der Jahresbeginn an, in die Zukunft zu blicken! 2011 scheint ein sehr «politisches» Jahr zu werden, mit einer Reihe von Debatten, die für uns Ärztinnen und Ärzte von grösster Bedeutung sind.

Dabei geht es um die integrierten Versorgungsnetze und den Risikoausgleich, aber auch um das Ende des Zulassungsstopps, die Einführung der DRG auf nationaler Ebene, die Zukunft der Krankenkassen, die Revision der Invalidenversicherung, das neue Präventionsgesetz und um weitere Fragen!

Die Position der FMH zu all diesen Themen beruht auf den «Strategischen Zielen 2008-2012» des Zentralvorstands, die von der Ärztekammer genehmigt wurden (siehe unter www.fmh.ch $\rightarrow$ FMH $\rightarrow$ Strategie). Die definierten Ziele widerspiegeln die bedeutende Rolle der Ärzteschaft bei politischen Entscheiden, die zentrale Stellung der Patientinnen und Patienten im Gesundheitssystem, die Qualität der Gesundheitsversorgung und der Ausbildung sowie die Gewährleistung unserer Lebens- und Arbeitsbedingungen.

\section{Die gegenwärtigen Strukturen der FMH ermöglichen eine effiziente und demokratische Zusammenarbeit.}

Auf der Basis dieser Leitlinien erörtert der Zentralvorstand anstehende politische Themen. Anschliessend befasst sich die Delegiertenversammlung damit und entscheidet über die Haltung der FMH.

Jeder politische Entscheid ergibt sich aus der Konfrontation und dem Ausgleich von zahlreichen, teilweise divergierenden Überlegungen. Doch dank den von der Ärztekammer klar gutgeheissenen strategischen Zielen und einem transparenten Entscheidungsprozess kann sowohl die Kohärenz unserer politischen Positionen als auch eine echte Vertretung der Auffassungen unserer Basis, d.h. von 35500 FMHMitgliedern (97\% der Schweizer Ärzteschaft!), gewährleistet werden. Die gegenwärtigen Strukturen der FMH ermöglichen eine effiziente und demokratische Zusammenarbeit.

Es ist jedoch eine gefährliche Illusion zu glauben, wir könnten parlamentarische, politische oder sogar gesellschaftliche Debatten blockieren, nur weil diese nicht unseren Vorstellungen entsprechen. Als Ärztinnen und Ärzte haben wir in der Gesellschaft eine sehr spezielle Stellung, dank der wir die erwähnten Debatten positiv beeinflussen können. Doch um uns Gehör zu verschaffen und unsere Interessen zu vertreten, müssen wir eine Sprache sprechen, die auch verstanden wird!

Die Modelle und unsere Ideale der letzten Jahrzehnte sind heutzutage unter Umständen weder verständlich noch angemessen: Deshalb stehen wir alle vor der schwierigen Aufgabe, das festzulegen, was unverrückbar sein muss, was für eine Medizin, die diesen Namen verdient, von zentraler Bedeutung ist und was an eine Gesellschaft angepasst werden muss, die sich auch ohne unser Dazutun weiterentwickelt. Ohne diese permanente Aktualisierung werden wir unsere Ziele nicht erreichen.

\section{Es ist eine gefährliche Illusion zu glauben, wir könnten Debatten blockieren, nur weil diese nicht unseren Vorstellungen entsprechen!}

In diesem Jahr werden wir das neue Präventionsgesetz vorbehaltlich überraschender Änderungen an der Endfassung unterstützen. Im Rahmen der IV-Revision setzen wir uns weiterhin engagiert für unsere Patientinnen und Patienten ein. Die Einführung der DRG verfolgen wir sehr aufmerksam, um allfällige Fehlentwicklungen verhindern oder rasch korrigieren zu können. Schliesslich werden wir dafür sorgen, dass unser Gesundheitssystem mit dem Ende des Zulassungsstopps nicht Schiffbruch erleidet, sondern bei Bedarf weiterhin gesteuert werden kann.

Im Bereich der integrierten Versorgungsnetze setzen wir uns weiterhin für einen Gesetzesentwurf ein, der wie der aktuell im Parlament beratene Entwurf zu einer bedeutenderen Rolle (und Verantwortung!) der Ärzteschaft bei der Organisation dieser Versorgungsnetze führt, aber keine kontraproduktive Verpflichtung wie die Budgetmitverantwortung umfasst. Dieser Gesetzesentwurf beinhaltet auch eine deutliche Verbesserung des Risikoausgleichs. Diesen werden wir engagiert unterstützen, weil er das einzige Mittel ist, um auf allen Ebenen unseres Gesundheitssystems eine Jagd nach guten Risiken zu verhindern - denn nur mit diesem verfeinerten Risikoausgleich kann sichergestellt werden, dass alle die benötigte Gesundheitsversorgung unter den bestmöglichen Bedingungen in Anspruch nehmen können.

Die Bedeutung der politischen Beteiligung der FMH an allen betreffenden Entscheidungen im Jahr 2011 kann nicht genug betont werden!

Für das nächste Jahr wünsche ich Ihnen allen viel Energie, Freude und Erfolg.

Dr. med. Jacques de Haller, Präsident der FMH 